\title{
Frailty, Inflammation, and Mortality Among Persons Aging With HIV Infection and Injection Drug Use
}

\section{Damani A. Piggott, ${ }^{1,2}$ Ravi Varadhan, ${ }^{3}$ Shruti H. Mehta, ${ }^{2}$ ToddT. Brown, ${ }^{1,2}$ Huifen Li, ${ }^{1}$ Jeremy D. Walston, ${ }^{1}$ Sean X. Leng, ${ }^{1}$ and Gregory D. Kirk ${ }^{1,2,3}$}

'Department of Medicine, Johns Hopkins University School of Medicine, Baltimore, Maryland. ${ }^{2}$ Department of Epidemiology, Johns Hopkins University School of Public Health, Baltimore, Maryland. ${ }^{3}$ Department of Oncology, Johns Hopkins University School of Medicine, Baltimore, Maryland.

Address correspondence to Damani A. Piggott, MD, PhD, Division of Infectious Diseases, Johns Hopkins University School of Medicine, 2213 McElderry Street, M141, Baltimore, MD 21205. Email: dpiggot1@jhmi.edu

Received December 24, 2014; Accepted June 12, 2015

Decision Editor: Stephen Kritchevsky, PhD

\begin{abstract}
Background. Serum markers of inflammation increase with age and have been strongly associated with adverse clinical outcomes among both HIV-infected and uninfected adults. Yet, limited data exist on the predictive and clinical utility of aggregate measures of inflammation. This study sought to evaluate the relationship of a recently validated aggregate inflammatory index with frailty and mortality among aging HIV-infected and uninfected injection drug users.

Methods. Frailty was assessed among HIV-infected and uninfected participants in the AIDS Linked to the IntraVenous Experience (ALIVE) cohort study using the five Fried phenotypic criteria: weight loss, exhaustion, low physical activity, decreased grip strength, and slow gait. The aggregate inflammatory index was constructed from serum measures of interleukin- 6 and soluble tumor necrosis factor$\alpha$ receptor-1. Multinomial logistic regression was used to assess the relationship of frailty with inflammation. Cox proportional hazards models were used to estimate risk for all-cause mortality. Results. Among 1,326 subjects, the median age was 48 years and $29 \%$ were HIV-infected. Adjusting for sociodemographics, comorbidity, and HIV status, frailty was significantly associated with each standard deviation increase in log interleukin-6 (odds ratio 1.33; 95\% Cl, 1.09-1.61), log tumor necrosis factor- $\alpha$ receptor-1 (odds ratio 1.25; $95 \% \mathrm{Cl}, 1.04-1.51$ ) and inflammatory index score (odds ratio $1.39 ; 95 \% \mathrm{Cl}$, 1.14-1.68). Adjusting for sociodemographics, comorbidity, HIV status, and frailty, the inflammatory index score was independently associated with increased mortality (HR 1.65; 95\% Cl, 1.44-1.89).

Conclusion. A recently validated, simple, biologically informed inflammatory index is independently associated with frailty and mortality risk among aging HIV-infected and uninfected injection drug users.
\end{abstract}

Key Words: Frailty—Inflammation—HIV—Mortality—Injection drug use

With the advent and increased uptake of combination antiretroviral therapy, HIV-infected persons are living longer $(1,2)$. Consequently, the proportion of older persons living with HIV infection has been increasing. This shift has been accompanied by an increasing burden of adverse age-associated outcomes including frailty and multimorbidity, along with persistent disparities in survival (3-6). Disparities 
in disease burden continue to be most severe for HIV-infected persons affected by injection drug use (6).

Frailty is an important aging-related state of increased vulnerability to internal and external stressors, considered the result of multisystem physiologic declines (7,8). Frailty has been strongly associated with major adverse clinical outcomes, including hospitalization, institutionalization, disability, and premature death. A frailty phenotype operationalized by Fried and colleagues has been commonly employed in the research literature and has been validated to predict adverse outcomes among older adults $(7,9,10)$. While first characterized predominantly among elderly populations (persons 65 years and older), the Fried frailty phenotype has more recently found applicability among aging though younger HIV-infected and uninfected populations. In these populations, the sociodemographic factors and clinical outcomes related to the frailty phenotype have been similar to those observed in the elderly (11-17).

Markers of inflammation increase with age and have been significantly associated with a range of prevalent and incident age-associated disease conditions (18-20). Heightened inflammation in particular has been strongly associated with premature mortality among elderly persons $(18,21,22)$. Dysregulated inflammatory pathways have also been central to HIV pathogenesis, and individual markers of inflammation have similarly been associated with age-associated disease and premature mortality in HIV-infected populations (23-28). Among elderly persons, the frailty phenotype has been consistently associated with heightened inflammation (29-32). Yet, the relationship of frailty with inflammation among notably younger, though aging, HIV-infected persons and their high risk counterparts has been less well defined.

Most analyses exploring the pathophysiologic role of inflammation among HIV-infected and elderly populations have focused on single markers of inflammation with few studies exploring aggregate measures of inflammation. Such aggregate measures have the potential to be of enhanced diagnostic, prognostic, and therapeutic utility. Recently, a biologically informed aggregate measure of inflammation was derived and validated to strongly and optimally capture the effect of inflammation on mortality among adults 65 years and older (33). This inflammatory index measure was derived from the assessment of 15 inflammatory markers regulated by the transcription factor nuclear factor kappa B in two well-established cohorts of elderly individuals - the Cardiovascular Health Study and InChianti. The predictive validity (hazard ratio) of this index for mortality in these cohorts was 1.62 (95\% CI, 1.54-1.70) and 1.33 (95\% CI, 1.17-1.52) respectively. The relevance of this aggregate inflammatory index to HIV-infected or younger populations is not known.

In prior studies, we demonstrated the significant association of the Fried frailty phenotype with HIV infection and mortality in a cohort of aging but with younger injection drug users (IDUs) (17). In this study, we sought to evaluate the relationship of the inflammatory index to the frailty phenotype in this cohort. We further sought to assess the relationship of the inflammatory index score to mortality in this younger population of HIV-infected and uninfected IDUs.

\section{Methods}

\section{Study Participants}

The AIDS Linked to the IntraVenous Experience (ALIVE) cohort has prospectively followed persons with a history of injecting drugs in a community-recruited cohort on a semiannual basis since 1988. IDUs aged 18 years or older were recruited through street-based efforts as previously detailed (34). The ALIVE study has been continually approved by the Johns Hopkins Institutional Review Board, and all participants provided written informed consent.

\section{Mortality Assessment}

Mortality was assessed through linkage to the National Death Index with review of death certificates to confirm correct matches.

\section{Frailty Assessment}

Frailty was assessed as previously described using the five original Fried criteria: slow gait, decreased grip strength (weakness), poor endurance (exhaustion), low physical activity, and physical shrinking (weight loss) (10,17). Each frailty parameter was considered as a binary variable $(0,1)$ and summed to obtain a frailty score; $\geq 3$ was considered frail, 1 or 2 considered prefrail, and scores of 0 considered robust.

\section{Inflammatory Index Assessment}

Interleukin-6 (IL-6) and soluble tumor necrosis factor receptor 1 (sTNFR1) were measured at a single study visit closest to the index frailty assessment. Testing was performed on serum samples collected and stored at $-80^{\circ} \mathrm{C}$ using commercially available enzyme-linked immunosorbent assay kits (R\&D Systems, Minneapolis, MN). IL-6 was measured using a High-Sensitivity Quantikine kit with a detection range of $0.156-10.0 \mathrm{pg} / \mathrm{mL}$ and interassay coefficient of variance of $5.7 \%$. sTNFR1 was measured using a DuoSet ELISA kit with a sensitivity of $12.5 \mathrm{pg} / \mathrm{mL}$ and an interassay coefficient of variance of $4.9 \%$. Measurements were performed in duplicate and repeated if the measures differed by more than $15 \%$ or were out of the measurable range. The average of the two measures was log transformed for these analyses to account for the nonnormal distribution of the individual inflammatory marker measures, as previously performed (33). The inflammatory index score was constructed from the IL-6 and sTNFR1 measurements as previously described [(1/3 log IL-6 + $2 / 3 \times \log$ sTNFR1)] (33).

\section{Other Measures}

Additional detailed information obtained at study visits included socioeconomic, behavioral, and clinical parameters for the prior 6 -month period. Comorbid conditions ascertained included obesity (defined as a body mass index $\geq 30$ ) and participant self-report of any provider diagnosis of diabetes, hypertension, or cerebrovascular, cardiovascular, renal, chronic lung, malignant, or liver disease. Depressive symptoms were assessed using the Center for Epidemiological Studies Depression Scale. Depressive symptomatology was defined as having a Center for Epidemiological Studies Depression Scale score of 21 or greater (17). Prescription drug abuse was by participant self-report of abuse of drugs prescribed to them by a physician in the last year.

Participants provided blood specimens for testing, which included antibodies to HIV-1 assayed by enzyme-linked immunosorbent assay, with Western blot confirmation. CD4 cell counts were measured on HIV-infected persons at each visit using flow cytometry, and plasma HIV-1 RNA levels were determined using reverse-transcriptase polymerase chain reaction methods.

\section{Statistical Analysis}

Multinomial logistic regression was employed to evaluate the crosssectional relationship of each individual inflammatory marker and the composite inflammatory index score with the frailty phenotype, 
treating frailty as a 3-tier categorical outcome. Models were adjusted for factors previously found or considered a priori to be associated with frailty, including age, gender, race, educational attainment, marital status, prescription drug abuse, depressive symptoms, number of comorbid disease conditions, and HIV status (17).

To evaluate the relationship between the inflammatory index score and all-cause mortality, Kaplan-Meier survival analyses and Cox proportional hazards regression models were performed. Observation was until date of death or for those remaining alive, December 31, 2010. Models were adjusted for factors previously found or considered a priori to be associated with mortality, including age, gender, race, educational attainment, number of comorbid disease conditions, and HIV status (17). The proportional hazards assumption was found to be reasonable by graphical assessment. Stratified analyses by HIV status were performed to assess the relationship of the inflammatory index with frailty and mortality independently among HIV-infected and HIV-uninfected participants respectively. Further, in sensitivity analyses, the relationship of the inflammatory index with the frailty phenotype and mortality risk was examined adjusting for CD4 cell count, HIV viral load, and use of combination antiretroviral therapy. Analyses were performed using STATA (version 12; Stata Corp., College Station, TX).

\section{Results}

Participant characteristics by frailty status are shown (Table 1 ). Among 1,326 participants, the median age was 48 years; $99 \%$ of participants were younger than 65 years; $88 \%$ were African American, 34\% were female, and 29\% were HIV-infected (Table 1). The median CD4 cell count for HIV-infected participants was 281 (interquartile range, 168-444) cells/ $\mu \mathrm{L}$, and the median HIV viral load was 3.15 (interquartile range, 1.6-4.5) $\log _{10}$ copies $/ \mathrm{mL}$. The median (interquartile range) levels of IL- 6 and sTNFR1 were 1.63 $(1.03,2.80) \mathrm{pg} / \mathrm{mL}$ and $1.51(1.26,1.86) \mathrm{ng} / \mathrm{mL}$, respectively. These levels were similar to those previously observed in older, HIVuninfected cohorts.

Univariate and multivariate associations of frailty with inflammation are shown in Table 2. In separate multivariable analyses adjusting for sociodemographics, depressive symptoms, number of comorbid disease conditions, and HIV status, frailty was significantly associated with each standard deviation increase in log IL-6 (odds ratio [OR] 1.33; 95\% CI, 1.09-1.61), log sTNFR1 (OR 1.25; 95\% CI, 1.04-1.51), and inflammatory index score (OR 1.39; $95 \%$ CI, 1.14-1.68) (Table 2). Further, in stratified analyses, frailty was significantly associated with each unit increase in the inflammatory index score for both HIV-infected (OR 1.53; 95\% CI, 1.04-2.26) and HIV-uninfected (OR 1.35; 95\% CI, 1.08-1.69) IDUs. No significant associations of the inflammatory index or its component markers were observed with prefrailty (Table 2 ).

During prospective evaluation of the relationship of the inflammatory index with mortality, we observed 144 deaths over 4,934 person-years for a mortality rate of 2.9 per 100 person-years. The median follow-up time was 4.3 years (interquartile range, 2.3-4.7) for both HIV-infected and HIV-uninfected participants. Overall, participants in the highest tertile of inflammation had substantially higher mortality compared with persons having lower inflammation; a dose-response increase in mortality was observed with increasing inflammation (Figure 1). In Cox proportional hazards models, adjusting for sociodemographic factors, number of comorbid disease conditions, HIV status, and frailty, the risk of mortality

Table 1. Baseline Characteristics of ALIVE Participants by Frailty Status*

\begin{tabular}{|c|c|c|c|}
\hline \multirow[t]{2}{*}{ Characteristics } & \multirow{2}{*}{$\begin{array}{l}\text { Robust } \\
N=338\end{array}$} & \multirow{2}{*}{$\begin{array}{l}\text { Prefrail } \\
N=826\end{array}$} & \multirow{2}{*}{$\begin{array}{l}\text { Frail } \\
N=162\end{array}$} \\
\hline & & & \\
\hline Age, median (IQR), y & $47.3(41.3-52.3)$ & $47.5(42.5-52.3)$ & $50.3(44.7-53.7)$ \\
\hline Female & $104(30.8 \%)$ & $285(34.5 \%)$ & $68(42 \%)$ \\
\hline African American & $296(87.6 \%)$ & $724(87.7 \%)$ & $144(88.9 \%)$ \\
\hline Less than high school education & $177(52.7 \%)$ & $494(59.9 \%)$ & $104(64.2 \%)$ \\
\hline Not married/common law & $303(90.2 \%)$ & $770(93.3 \%)$ & $152(93.8 \%)$ \\
\hline Prescription drug abuse ${ }^{\dagger}$ & $25(7.4 \%)$ & $110(13.3 \%)$ & $27(16.7 \%)$ \\
\hline Recent injection drug use $\mathrm{e}^{\ddagger}$ & $164(48.5 \%)$ & $366(44.3 \%)$ & $73(45.1 \%)$ \\
\hline Depressive symptoms $\mathrm{s}^{\ddagger}$ & $48(14.2 \%)$ & $201(24.3 \%)$ & $71(43.8 \%)$ \\
\hline \multicolumn{4}{|l|}{ \# Comorbid conditions ${ }^{\S}$} \\
\hline $0-1$ & $262(78.4 \%)$ & $605(75.1 \%)$ & $98(62.8 \%)$ \\
\hline 2 & $50(15 \%)$ & $124(15.4 \%)$ & $27(17.3 \%)$ \\
\hline$\geq 3$ & $22(6.6 \%)$ & $77(9.6 \%)$ & $31(19.9 \%)$ \\
\hline HIV seropositive & $86(25.4 \%)$ & $244(29.5 \%)$ & $57(35.2 \%)$ \\
\hline $\mathrm{CD} 4{ }^{+}$cell count, median $(\mathrm{IQR})^{\|}$ & $318(185-486)$ & $262(158-420)$ & $312(145-505)$ \\
\hline HIV RNA, median (IQR), $\log _{10}$ copies/mL" & $2.7(1.6-4.4)$ & $3.2(1.6-4.6)$ & $3.7(1.8-4.7)$ \\
\hline IL-6 (pg/mL), median (IQR) & $1.44(0.98-2.37)$ & $1.64(1.03-2.96)$ & $1.90(1.31-3.60)$ \\
\hline sTNFR1 (ng/mL), median (IQR) & $1.48(1.25-1.79)$ & $1.50(1.26-1.85)$ & $1.65(1.36-2.06)$ \\
\hline Inflammatory index score, mean $(S D)$ & $5.07(0.43)$ & $5.12(0.47)$ & $5.28(0.54)$ \\
\hline
\end{tabular}

Notes: Depressive symptoms $=$ score of $\geq 21$ on the Center for Epidemiological Studies Depression Scale; IL-6 = interleukin-6; IQR = interquartile range; sTNFR1 = soluble tumor necrosis factor- $\alpha$ receptor- 1 .

*Data are number $(\%)$ of participants, unless otherwise indicated; robust participants had a frailty score of 0; prefrail participants had a frailty score of 1-2; frail participants had a frailty score of 3-5.

${ }^{\dagger}$ Reflect characteristics within the prior year.

${ }^{\ddagger}$ Reflect characteristics within the previous 6 months.

Diabetes, hypertension, cerebrovascular accident, cardiovascular disease, renal disease, chronic obstructive pulmonary disease, cancer, obesity, liver disease.

"Reflect HIV seropositives only. 
independently increased with each standard deviation increase in log IL-6 (HR 1.38; 95\% CI, 1.19-1.59), log sTNFR1 (HR 1.54; 95\% CI, 1.39-1.71), and the inflammatory index score (HR 1.65; $95 \%$ CI, 1.44-1.89). Independent of the inflammatory index score, the frailty phenotype remained a significant predictor of mortality risk in multivariate models (HR 2.07; 95\% CI, 1.17-3.65); the interaction term for the effect of frailty and the inflammatory index score on mortality was not significant $(p=.4)$. In multivariate analysis stratified by HIV status (Table 3), the association of the inflammatory index with mortality was similar for HIV-infected (HR 1.55; 95\% CI, 1.26-1.91) and HIV-uninfected (HR 1.61; 95\% CI, 1.35$1.92)$ persons. In sensitivity analyses adjusting for active injection drug use, there was no difference in the relationship of the inflammatory index score with either frailty or mortality. Further, when we used more refined estimates of HIV clinical parameters the risk estimates for the relationship of the inflammatory index with both frailty and mortality were unchanged (Supplementary Figure 1).

\section{Discussion}

We show that a recently validated, simple, biologically informed inflammatory index is strongly associated with frailty and independently associated with mortality among aging HIV-infected and uninfected IDUs. This index was a stronger predictor of premature death than its individual components. Extended beyond the older general population, this study demonstrates the applicability of an aggregate measure of inflammation to evaluating key aging-related outcomes among younger but aging HIV-infected and high risk populations. Our data suggest similarities in the biological processes that promote frailty in HIV-infected and elderly populations. Moreover, it contributes to the increasing evidence base on the key role of inflammatory pathways in driving adverse aging-related outcomes in HIV-infected populations and their uninfected high risk counterparts.

Persistently heightened inflammation has been considered a central feature of both pathological aging and chronic HIV disease. We previously demonstrated the independent associations of age and HIV infection with inflammation, as well as separately demonstrated the association of age and HIV infection with frailty $(17,35)$. We also previously demonstrated the combined deleterious effect of HIV infection and frailty on premature death (17). Our findings here suggest that the heightened mortality associated with being both HIV-infected and frail may in part be driven by the combined effects of HIV-associated and frailty-associated inflammation, likely reflecting the intersecting biology shared by HIV-related and pathological aging processes.

There have been multiple proposed drivers of heightened inflammation among aging and HIV-infected persons. Such pathways include immune activation, microbial translocation, immunosenescence, cytomegalovirus replication, and loss of immunoregulation $(23,25,28,36)$. Further exploration of the role of these biological

Table 2. Odds of Frailty and Prefrailty by Level of Inflammation in ALIVE*

\begin{tabular}{|c|c|c|c|c|}
\hline \multirow[t]{3}{*}{ Model } & Prefrail & Prefrail & Frail & Frail \\
\hline & Unadjusted & Adjusted $^{\dagger}$ & Unadjusted & Adjusted $^{\dagger}$ \\
\hline & OR $(95 \% \mathrm{CI})$ & OR $(95 \% \mathrm{CI})$ & OR $(95 \% \mathrm{CI})$ & OR $(95 \% \mathrm{CI})$ \\
\hline IL-6 & $1.13(0.99-1.29)$ & $1.09(0.95-1.26)$ & $1.40(1.17-1.68)$ & $1.33(1.09-1.61)$ \\
\hline sTNFR1 & $1.08(0.93-1.24)$ & $1.03(0.89-1.19)$ & $1.40(1.17-1.66)$ & $1.25(1.04-1.51)$ \\
\hline Inflammatory index score & $1.13(0.99-1.30)$ & $1.08(0.94-1.25)$ & $1.53(1.28-1.83)$ & $1.39(1.14-1.68)$ \\
\hline
\end{tabular}

Notes: IL-6, interleukin-6; sTNFR1, soluble tumor necrosis factor- $\alpha$ receptor 1.

*Data are given as unadjusted and adjusted odds ratios (95\% confidence interval)—increase in the odds ratio for being prefrail (compared to robust) and for being frail (compared to robust), per standard deviation increase in inflammatory marker. Robust participants had a frailty score of 0 ; prefrail participants had a frailty score of 1-2; frail participants had a frailty score of 3-5.

${ }^{\dagger}$ Each model was adjusted for age, gender, race, education, marital status, prescription drug abuse, depressive symptoms, \# comorbid conditions and HIV status as per prior analyses (17).

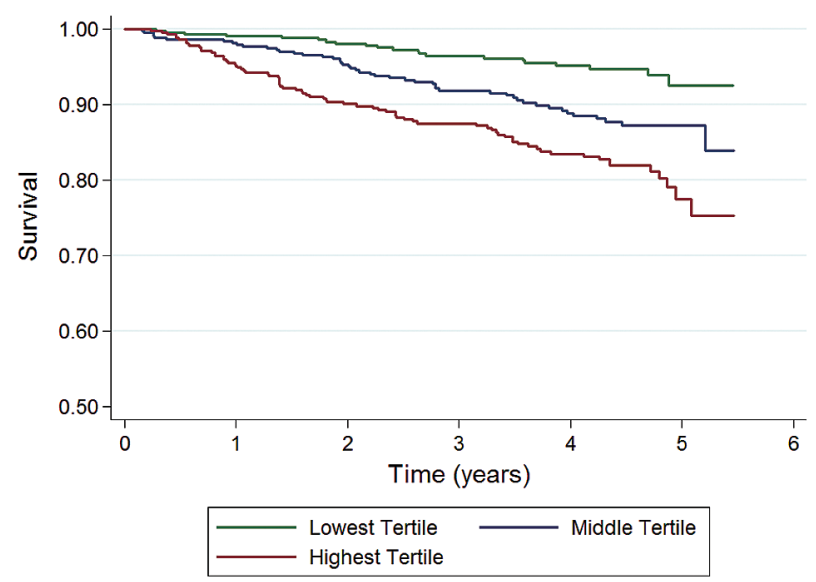

Figure 1. Survival by tertile of inflammatory index in the ALIVE cohort. Kaplan-Meier survival curve estimates by tertile of inflammatory index score.
Table 3. Mortality Risk Associated With the Inflammatory Index Among ALIVE Participants*

\begin{tabular}{|c|c|c|}
\hline & Unadjusted HR $\mathrm{H}^{\S}$ & Adjusted HR ${ }^{\S}$ \\
\hline & $(95 \% \mathrm{CI})$ & $(95 \% \mathrm{CI})$ \\
\hline All participants ${ }^{\dagger}$ & $1.79(1.58-2.02)$ & $1.65(1.44-1.89)$ \\
\hline HIV-uninfected only ${ }^{\ddagger}$ & $1.91(1.61-2.25)$ & $1.61(1.35-1.92)$ \\
\hline HIV-infected only ${ }^{\ddagger}$ & $1.61(1.33-1.94)$ & $1.55(1.26-1.91)$ \\
\hline
\end{tabular}

Notes: HR = hazard ratio

*Data are given as unadjusted and adjusted hazard ratios $(95 \%$ confidence interval).

${ }^{\dagger}$ Adjusted for age, gender, race, education, \# comorbid conditions, HIV status, and frailty status.

‡Adjusted for age, gender, race, education, \# comorbid conditions, and frailty status.

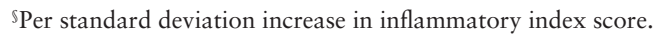


factors in promoting adverse aging-related outcomes such as frailty and premature mortality among HIV-infected persons and their high risk counterparts is needed.

As the putative role of inflammation in driving adverse agingrelated outcomes among HIV-infected persons gains support, increasing focus has been placed on developing and evaluating antiinflammation strategies to prevent or reverse pathological aging processes. Novel therapeutic modalities may potentially be derived from research on the biological processes driving inflammation. However, existing antiretroviral therapy (ART) and non-ART approaches to reduce inflammation could potentially be employed to improve outcomes among HIV-infected persons.

Effective ART is known to reduce inflammation among HIVinfected persons $(23,27)$. Earlier ART has been associated with enhanced CD4 recovery along with a reduced inflammatory state (37). Greater efforts at both increased and earlier uptake of ART, particularly for those co-affected by substance use, may potentially reduce inflammation-mediated damage and prevent adverse outcomes (38-40).

However, early and effective ART alone may not suffice to eliminate the negative impact of inflammation on HIV-infected populations. Persistent heightened inflammation has been observed for many HIV-infected persons on effective ART, even with reduced viremia. In addition, behavioral factors known to be of high burden among HIV-infected populations (eg, tobacco use, injection drug use) have also been associated with heightened inflammation $(18,41)$. Thus, efforts including both pharmacotherapeutic approaches and lifestyle interventions are needed to address non-HIV specific factors associated with inflammation $(18,27)$.

While mortality risk for both frailty and inflammation was attenuated in multivariate models, frailty and inflammation remained independently associated with mortality in this study. Inflammation, while increasingly identified as a central precipitant of adverse outcomes in the aging HIV-infected population, likely represents a complex component of more multifaceted aging-related pathways. In elderly HIV-uninfected populations, additional biological pathways have been linked to frailty and other aging pathophysiology, including neuroendocrine dysfunction, oxidative stress, and aberrant coagulation pathways $(36,42-45)$. The relevance of these pathways to frailty and other adverse aging-related outcomes for HIV-infected populations requires further investigation and may serve as additional putative targets for intervention.

The inflammation-related markers used to construct the aggregate index were selected a priori based on knowledge of nuclear factor kappa B-related biology (33). There may be aspects of the inflammation cascade not captured by this index that would be relevant to aging-related outcomes in HIV-infected persons. Given the cross-sectional assessment of frailty and concurrent inflammation in this study, inferences regarding causality are not possible. Heightened inflammation has been associated with disability (18). Disability measures were not available for the study. However, this study was conducted in an ambulatory cohort, with a lower likelihood of disability effects. Nevertheless, as the prevalence of disability in this population increases with age, further study of the complex relationship observed between frailty and disability in HIVuninfected elderly populations will be warranted (7). Further, this cohort was a predominantly African American, urban IDU cohort of lower socioeconomic status and, as such, our findings may not be fully generalizable to other HIV-infected populations. However, the cohort represents those persons most adversely affected by the HIV epidemic and those most subject to disparities in adverse agingrelated outcomes $(6,46)$. Moreover, the inflammation index was initially validated in two predominantly white, more highly educated cohorts of persons aged 65 years and older. This study extends the utility of this index to younger, minority populations of lower socioeconomic status with and without comorbid HIV infection.

In summary, this study demonstrates the significant relationship between a simple, biologically informed aggregate index of inflammation with both frailty and mortality among HIV-infected persons and their high risk uninfected counterparts. Our findings highlight the need for further investigation of inflammatory pathways in developing interventions to promote healthy aging. Studies incorporating the inflammation index, the frailty phenotype, and other key aging-related measures in older, HIV-infected persons and their high risk HIV-uninfected counterparts may help elucidate the underlying biology of the aging process in HIV.

\section{Supplementary Material}

Supplementary material can be found at: http://biomedgerontology. oxfordjournals.org/

\section{Funding}

This work was supported by the National Institute of Allergy and Infectious Diseases and the National Institute on Drug Abuse at the National Institutes of Health (K23-AI-108357, RC1-AI-086053, U01-DA-023832, RO1-DA-12568).

\section{Acknowledgments}

The authors would like to thank the ALIVE study participants and ALIVE study staff for their significant contributions to this work.

\section{References}

1. High KP, Brennan-Ing M, Clifford DB, et al. HIV and aging: state of knowledge and areas of critical need for research. A report to the NIH Office of AIDS Research by the HIV and Aging Working Group. J Acquir Immune Defic Syndr. 2012;60(suppl 1):S1-S18. doi:10.1097/ QAI.0b013e31825a3668

2. Mills EJ, Bärnighausen T, Negin J. HIV and aging-preparing for the challenges ahead. N Engl J Med. 2012;366:1270-1273. doi:10.1056/ NEJMp1113643

3. Brothers TD, Rockwood K. Biologic aging, frailty, and age-related disease in chronic HIV infection. Curr Opin HIV AIDS. 2014;9:412-418. doi:10.1097/COH.0000000000000070

4. Guaraldi G, Orlando G, Zona S, et al. Premature age-related comorbidities among HIV-infected persons compared with the general population. Clin Infect Dis. 2011;53:1120-1126. doi:10.1093/cid/cir627

5. Salter ML, Lau B, Go VF, Mehta SH, Kirk GD. HIV infection, immune suppression, and uncontrolled viremia are associated with increased multimorbidity among aging injection drug users. Clin Infect Dis. 2011;53:1256-1264. doi:10.1093/cid/cir673

6. Samji H, Cescon A, Hogg RS, et al. Closing the gap: increases in life expectancy among treated HIV-positive individuals in the United States and Canada. PLoS One. 2013;8:e81355. doi:10.1371/journal.pone.0081355

7. Fried LP, Ferrucci L, Darer J, Williamson JD, Anderson G. Untangling the concepts of disability, frailty, and comorbidity: implications for improved targeting and care. J Gerontol A Biol Sci Med Sci. 2004;59:M255-M263. doi:10.1093/gerona/59.3.M255

8. Xue QL. The frailty syndrome: definition and natural history. Clin Geriatr Med.2011;27:1-15. doi:10.1016/j.cger.2010.08.009

9. Bandeen-Roche K, Xue QL, Ferrucci L, et al. Phenotype of frailty: characterization in the women's health and aging studies. J Gerontol A Biol Sci Med Sci. 2006;61:262-266. 
10. Fried LP, Tangen CM, Walston J, et al. Frailty in older adults: evidence for a phenotype. J Gerontol A Biol Sci Med Sci. 2001;56:M146-M157. doi:10.1093/gerona/56.3.M146

11. Althoff KN, Jacobson LP, Cranston RD, et al. Age, comorbidities, and AIDS predict a frailty phenotype in men who have sex with men. J Gerontol A Biol Sci Med Sci. 2014;69:189-198. doi:10.1093/gerona/glt148

12. Desquilbet L, Jacobson LP, Fried LP, et al. HIV-1 infection is associated with an earlier occurrence of a phenotype related to frailty. J Gerontol A Biol Sci Med Sci. 2007;62:1279-1286.

13. Erlandson KM, Allshouse AA, Jankowski CM, et al. Comparison of functional status instruments in HIV-infected adults on effective antiretroviral therapy. HIV Clin Trials. 2012;13:324-334. doi:10.1310/hct1306-324

14. McAdams-DeMarco MA, Law A, Salter ML, et al. Frailty as a novel predictor of mortality and hospitalization in individuals of all ages undergoing hemodialysis. J Am Geriatr Soc. 2013;61:896-901. doi:10.1111/jgs.12266

15. Onen NF, Agbebi A, Shacham E, Stamm KE, Onen AR, Overton ET. Frailty among HIV-infected persons in an urban outpatient care setting. $J$ Infect. 2009;59:346-352. doi:10.1016/j.jinf.2009.08.008

16. Pathai S, Gilbert C, Weiss HA, et al. Frailty in HIV-infected adults in South Africa. J Acquir Immune Defic Syndr. 2013;62:43-51. doi:10.1097/ QAI.0b013e318273b631

17. Piggott DA, Muzaale AD, Mehta SH, et al. Frailty, HIV infection, and mortality in an aging cohort of injection drug users. PLoS One. 2013;8:e54910. doi:10.1371/journal.pone. 0054910

18. Singh T, Newman AB. Inflammatory markers in population studies of aging. Ageing Res Rev. 2011;10:319-329. doi:10.1016/j.arr.2010.11.002

19. Fabbri E, An Y, Zoli M, et al. Aging and the burden of multimorbidity: associations with inflammatory and anabolic hormonal biomarkers. J Gerontol A Biol Sci Med Sci. 2015;70:63-70. doi:10.1093/gerona/glu127

20. Franceschi C, Campisi J. Chronic inflammation (inflammaging) and its potential contribution to age-associated diseases. J Gerontol A Biol Sci Med Sci. 2014;69(suppl 1):S4-S9. doi:10.1093/gerona/glu057

21. Newman AB, Sachs MC, Arnold AM, et al. Total and cause-specific mortality in the cardiovascular health study. J Gerontol A Biol Sci Med Sci. 2009;64:1251-1261. doi:10.1093/gerona/glp127

22. Roubenoff R, Parise H, Payette HA, et al. Cytokines, insulin-like growth factor 1, sarcopenia, and mortality in very old community-dwelling men and women: the Framingham Heart Study. Am J Med. 2003;115:429-435.

23. Deeks SG. HIV infection, inflammation, immunosenescence, and aging. Annu Rev Med. 2011;62:141-155. doi:10.1146/annurev-med-042909-093756

24. Erlandson KM, Allshouse AA, Jankowski CM, et al. Association of functional impairment with inflammation and immune activation in HIV type 1-infected adults receiving effective antiretroviral therapy. J Infect Dis. 2013;208:249-259. doi:10.1093/infdis/jit147

25. Hearps AC, Martin GE, Rajasuriar R, Crowe SM. Inflammatory co-morbidities in HIV+ individuals: learning lessons from healthy ageing. Curr HIV/AIDS Rep. 2014;11:20-34. doi:10.1007/s11904-013-0190-8

26. Kuller LH, Tracy R, Belloso W, et al. Inflammatory and coagulation biomarkers and mortality in patients with HIV infection. PLoS Med. 2008;5:e203. doi:10.1371/journal.pmed.0050203

27. Sandler NG, Sereti I. Can early therapy reduce inflammation? Curr Opin HIV AIDS. 2014;9:72-79. doi:10.1097/COH.0000000000000020

28. Pathai S, Bajillan H, Landay AL, High KP. Is HIV a model of accelerated or accentuated aging? J Gerontol A Biol Sci Med Sci. 2014;69:833-842. doi:10.1093/gerona/glt168

29. Collerton J, Martin-Ruiz C, Davies K, et al. Frailty and the role of inflammation, immunosenescence and cellular ageing in the very old: cross-sectional findings from the Newcastle 85+ Study. Mech Ageing Dev. 2012;133:456-466. doi:10.1016/j.mad.2012.05.005
30. Hubbard RE, O'Mahony MS, Savva GM, Calver BL, Woodhouse KW. Inflammation and frailty measures in older people. J Cell Mol Med. 2009;13:3103-3109. doi:10.1111/j.1582-4934.2009.00733.x

31. Leng SX, Xue QL, Tian J, Walston JD, Fried LP. Inflammation and frailty in older women. J Am Geriatr Soc. 2007;55:864-871. doi:10.1111/j.15325415.2007.01186.x

32. Walston J, McBurnie MA, Newman A, et al.; Cardiovascular Health Study. Frailty and activation of the inflammation and coagulation systems with and without clinical comorbidities: results from the Cardiovascular Health Study. Arch Intern Med. 2002;162:2333-2341. doi:10.1001/ archinte.162.20.2333

33. Varadhan R, Yao W, Matteini A, et al. Simple biologically informed inflammatory index of two serum cytokines predicts 10 year all-cause mortality in older adults. J Gerontol A Biol Sci Med Sci. 2014;69:165-173. doi:10.1093/gerona/glt023

34. Vlahov D, Anthony JC, Munoz A, et al. The ALIVE study, a longitudinal study of HIV-1 infection in intravenous drug users: description of methods and characteristics of participants. NIDA Res Monogr. 1991;109:75-100.

35. Salter ML, Lau B, Mehta SH, Go VF, Leng S, Kirk GD. Correlates of elevated interleukin- 6 and C-reactive protein in persons with or at high risk for HCV and HIV infections. J Acquir Immune Defic Syndr. 2013;64:488495. doi:10.1097/QAI.0b013e3182a7ee2e

36. Fedarko NS. The biology of aging and frailty. Clin Geriatr Med. 2011;27:27-37. doi:10.1016/j.cger.2010.08.006

37. Le T, Wright EJ, Smith DM, et al. Enhanced CD4+ T-cell recovery with earlier HIV-1 antiretroviral therapy. N Engl J Med. 2013;368:218-230. doi:10.1056/NEJMoa1110187

38. Gardner EM, McLees MP, Steiner JF, Del Rio C, Burman WJ. The spectrum of engagement in HIV care and its relevance to test-and-treat strategies for prevention of HIV infection. Clin Infect Dis. 2011;52:793800. doi:10.1093/cid/ciq243

39. Kavasery R, Galai N, Astemborski J, et al. Nonstructured treatment interruptions among injection drug users in Baltimore, MD. J Acquir Immune Defic Syndr. 2009;50:360-366. doi:10.1097/QAI.0b013e318198a800

40. Westergaard RP, Hess T, Astemborski J, Mehta SH, Kirk GD. Longitudinal changes in engagement in care and viral suppression for HIVinfected injection drug users. AIDS. 2013;27:2559-2566. doi:10.1097/ QAD.0b013e328363bff2

41. Kirk GD, Merlo CA; Lung HIV Study. HIV infection in the etiology of lung cancer: confounding, causality, and consequences. Proc Am Thorac Soc. 2011;8:326-332. doi:10.1513/pats.201009-061WR

42. Walston J, Hadley EC, Ferrucci L, et al. Research agenda for frailty in older adults: toward a better understanding of physiology and etiology: summary from the American Geriatrics Society/National Institute on Aging Research Conference on Frailty in Older Adults. J Am Geriatr Soc. 2006;54:991-1001. doi:10.1111/j.1532-5415.2006.00745.x

43. Roubenoff R. The "cytokine for gerontologists" has some company. J Gerontol A Biol Sci Med Sci. 2014;69:163-164. doi:10.1093/gerona/glt184

44. Sanders JL, Ding V, Arnold AM, et al. Do changes in circulating biomarkers track with each other and with functional changes in older adults? J Gerontol A Biol Sci Med Sci. 2014;69:174-181. doi:10.1093/gerona/ glt088

45. Schöttker B, Saum KU, Jansen EH, et al. Oxidative stress markers and allcause mortality at older age: a population-based cohort study. J Gerontol A Biol Sci Med Sci. 2015;70:518-524. doi:10.1093/gerona/glu111

46. Simard EP, Fransua M, Naishadham D, Jemal A. The influence of sex, race/ ethnicity, and educational attainment on human immunodeficiency virus death rates among adults, 1993-2007. Arch Intern Med. 2012;172:15911598. doi:10.1001/archinternmed.2012.4508 